\title{
Identifying Prognostic Factors of Severe Metabolic Acidosis and Acute Kidney Injury in African Children with Severe Falciparum Malaria: A Secondary Analysis of A Randomised Trial
}

\section{Grace Wezi Mzumara ( $\nabla$ gmzumara@mlw.mw)}

Malawi-Liverpool-Wellcome Trust Clinical Research Programme https://orcid.org/0000-0001-81321620

Stije Stije Leopold

University of Oxford

Kevin Marsh

Oxford University: University of Oxford

Arjen Dondorp

Mahidol Oxford Tropical Medicine Research Unit

Eric Ohuma

London School of Hygiene \& Tropical Medicine

Mavuto Mukaka

Mahidol Oxford Tropical Medicine Research Unit

\section{Research}

Keywords: Severe Malaria, Metabolic acidosis, Acute Kidney injury, Plasmodium Falciparum, Africa

Posted Date: January 19th, 2021

DOl: https://doi.org/10.21203/rs.3.rs-148298/v1

License: (a) (i) This work is licensed under a Creative Commons Attribution 4.0 International License. Read Full License 


\section{Abstract}

\section{Introduction}

Severe metabolic acidosis and acute kidney injury are major causes of mortality in children with severe malaria but are often underdiagnosed in low resource settings.

\section{Methods}

We conducted a retrospective analysis of the 'Artesunate vs Quinine in the treatment of severe falciparum malaria in African children' (AQUAMAT) trial to identify clinical features of severe metabolic acidosis and acute kidney injury in 5425 children from nine African countries. Separate models were fitted for acute kidney injury and severe metabolic acidosis. Separate univariable and multivariable logistic regression were performed to identify prognostic factors for severe metabolic acidosis (SMA) and acute kidney injury (AKI). Both analyses adjusted for the trial arm. A forward selection approach was used for model building of the logistic models and a threshold of $5 \%$ statistical significance was used for inclusion of variables into the final logistic model. Model performance was assessed through calibration, discrimination, and internal validation with bootstrapping.

\section{Results}

There were 2296 children identified with Severe metabolic acidosis and 1110 with Acute Kidney Injury. Prognostic features of SMA among them were: deep breathing (OR: 5.41, Cl: $4.26-6.89$ ), hypoglycaemia (OR: 5.22, Cl: 3.80 - 7.18), AKI (OR: 3.99, Cl: $3.30-4.81$ ), coma ( OR: 1.79 Cl: 1.36 2.35), respiratory distress (OR: 1.49, Cl: $1.21-1.83$ ), prostration (OR: $1.64 \mathrm{Cl}: 1.30-2.03$ ) and severe anaemia (OR: 1.40, Cl: 1.11 - 1.77). Features associated with AKI were; older children(OR: 1.20, Cl: 1.15 1.25), coma (2.47, Cl: 1.78 - 3.42), Prostration (OR: $1.52 \mathrm{Cl}: 1.14$ - 2.02), decompensated shock (OR: 1.74, Cl: 1.15 - 2.63), black water fever (Cl: 1.81. Cl: 1.22 - 2.69), jaundice (OR: $3.31 \mathrm{Cl}: 2.01-5.47)$, SMA (OR: $4.02 \mathrm{Cl} 3.30$ - 4.89), mild anaemia (OR: 1.36, Cl: 1.05 - 1.76), severe anaemia (OR: 1.48, Cl: 1.11 - 1.96), hypoglycaemia (OR: 2.02, Cl: $1.58-2.59$ ), hypernatremia (OR: $5.74, \mathrm{Cl}: 2.69-12.26$ ) and hyperkalaemia (OR: 5.31 . Cl: $4.15-6.80$ ).

\section{Conclusion}

Clinical and laboratory parameters representing contributors and consequences of severe metabolic acidosis and acute kidney injury were independently associated with these outcomes. The model can be useful for identifying patients at high risk of these complications where laboratory assessments are not routinely available.

\section{Introduction}

A major challenge to preventing mortality from Malaria is early identification and mitigation of clinical states predictive of death from malaria. The WHO Africa region bears the brunt as Malaria mortality 
accounts for $86 \%$ of global malaria cases, and $80 \%$ of all malaria related deaths (1). Despite prompt administration of anti-malaria's, $5.8 \%-24 \%$ of childhood deaths from malaria will occur on the first day of admission (2,3). Metabolic acidosis and acute kidney injury are two of three (in addition to coma) complications of Malaria most predictive for a fatal outcome of severe malaria $(2,4-10)$. However, diagnosis of metabolic acidosis or acute kidney injury requires laboratory facilities beyond the basic test facilities often available. Assessing the base excess to quantify metabolic acidosis, requires a blood gas machine, and also assessing plasma lactate, which can be a surrogate maker for acidosis, is often not routinely available. Acute renal injury can be identified rapidly if the patient with severe malaria is anuric. However, up to $80 \%$ of severe malaria patients with Acute Kidney Injury (AKI) are non-oliguric (11-13). Identifying these children with a high risk of having AKI could be beneficial in resource-poor settings.

In children with severe malaria, metabolic acidosis is present in up to $50 \%$ of cases, and is strongly associated with a fatal outcome $(10,14,15)$. Metabolic acidosis in Malaria is mainly a result of increased lactate (LA) production from anaerobic glycolysis and to a smaller extent from $P$. falciparum parasites (5, 8). The plasma and urinary acids $a$-hydroxybutyric acid (aHBA), $\beta$-hydroxybutyric acid ( $\beta H B A$ ), and $p$ hydroxyphenyllactic acid (HPLA), also contribute to the metabolic acidosis $(5-7,16)$. Failure of hepatic gluconeogenesis and lack of renal clearance due to acute kidney injury commonly compound this problem $(8,16)$. These biochemical changes cause physiologic responses and clinical signs that can be used to identify metabolic acidosis in patients.

The clinical signs associated with metabolic acidosis are varied and there are no reliable criteria for making this diagnosis clinically. Although deep breathing is a sign of respiratory distress most commonly described in relation to metabolic acidosis, its association has been contested in several studies $(2,14$, $17,18)$. Similarly, there is varied significance in the association of metabolic acidosis and the presence of coma or convulsions $(17,19)$. Due to the overlapping nature of these and other clinical signs, it is challenging to identify metabolic acidosis clinically (20).

In children, acute kidney injury is underdiagnosed and presents a growing concern for renal disease as a global health problem in Africa (21). In Nigeria, Malaria was the third commonest cause of AKI among children admitted to a nephrology unit (13). Among Congolese children in intensive care, black water fever from malaria was the predominant cause of AKI (12). In Uganda, nearly half (45.5\%) of children with severe malaria had Kidney Disease Improving Global Outcomes defined AKI (21). Challenges in diagnosis AKI in resource limited settings mean that it is underdiagnosed consequently undertreated, leading to increasing mortality $(4,10,21,22)$.

Having reliable surrogate markers that identify metabolic acidosis and AKI could improve their diagnosis. However, studies on clinical signs of severe metabolic acidosis and AKI in malaria often have low sensitivity and high inter-observer variability $(18,20,23)$. Reliably identifying these complications of malaria would improve the efficiency of therapeutic approaches within the first 24 hours of admission.

The aim of this study was to develop prognostic models for identification of factors related to severe metabolic acidosis and acute kidney injury using clinical signs among children admitted with severe 
malaria.

\section{Methods}

\section{Study design, study population and sampling}

This is a secondary analysis of the open label Artesunate Versus Quinine in the Treatment of severe falciparum malaria in African children randomised controlled trial (AQUAMAT) in eleven centres of nine African countries (24). The main aim of the study was to compare mortality outcomes in the Quinine and Artesunate arms of the trial. Selected patients had a positive blood antigen dipstick test for Plasmodium Falciparum histidine rich protein2, and a physician's clinical diagnosis of severe malaria. Severe malaria was defined as patients with a positive malaria test and at least one of the WHO symptoms (24). Patients with previous treatment of Quinine (40 mg/kg on first day and $30 \mathrm{mg} / \mathrm{kg}$ on any subsequent day) or Artesunate derivatives within 24 hours preceding admission to the study were excluded (24).

The AQUAMAT randomised trial enrolled 5425 children from 11 centres in nine African countries between October 2005 and July 2010. The participating countries and their number of participants are: The Gambia (502, 9.3\%), Ghana (436, 8\%), Kenya (442, 8.3\%), Tanzania (1461, 26.9\%), Nigeria (450, 8.3\%), Uganda (663, 12.2\%), Mozambique (664, 12.2\%), Rwanda (386, 7.1\%), and Democratic Republic of the Congo (422, 7.8\%) (24). There were 2713 patients in the Artesunate arm and 2713 patients in the Quinine arm analysed by intention to treat (24). Overall mortality was 9.7\% (527), with 230 (8.5\%) deaths in the Artesunate group and 297 (10.9\%) deaths in the Quinine group (24). The study supported adopting Artesunate as the first line of treatment for plasmodium falciparum Malaria in children. Other analyses of mortality findings have been discussed in secondary analyses of the AQUAMAT study $(4,24)$.

Definitions of Model Outcomes and Candidate Predictors

We searched literature on severe metabolic acidosis and AKI in children with severe malaria to identify features associated with these complications that were studied or previously described (Fig. 1). We included candidate predictors that were complications of malaria and those that either caused or resulted from severe metabolic acidosis or AKI. For severe metabolic acidosis, this includes contributing factors like anaemia and consequences of metabolic acidosis, like deep breathing $(17,25)$. Similarly, for acute kidney injury, candidate predictors include causative features like black water fever and hypovolaemia $(26,27)$. Our candidate predictors also included laboratory features important to severe malaria such as sodium and potassium measurements.

The outcome and candidate predictor cut-off points were selected according to WHO classifications for severe metabolic acidosis and acute kidney injury (28). Accordingly, we defined severe metabolic acidosis as a base excess of less than $-8 \mathrm{mmol} / \mathrm{l}$ (28). This cut-off for base excess has also been used in studies in similar settings $(14,20)$. Similarly, AKI was defined as a Blood Urea Nitrogen (BUN) of more than $20 \mathrm{mg} / \mathrm{dl}$ (28). Candidate predictors for each outcome were selected based on WHO signs of severe malaria and variables described in literature to be associated with each outcome. 
Figure 1: Conceptual Framework of Features associated with severe metabolic acidosis and acute kidney injury in children with Severe Falciparum Malaria (29)

\section{Statistical analysis}

We used the forward selection for inclusion of predictors for the diagnosis of severe metabolic acidosis and acute kidney injury. To select candidate predictors for each outcome, a univariable logistic regression was used and a 5\% significance level threshold for inclusion of variables into the model using forward selection approach.

Key predictors and some selected patient demographics were included in the multivariable logistic regression analysis irrespective of statistical significance. The multivariable model was adjusted for trial arm (quinine and artesunate) and the final model included only variables that were statistically significant.

The performance of the predictive models was assessed through calibration and discrimination. A calibration plot of predicted observed against predicted events was plotted for 10 equal groups to measure the agreement in probabilities of developing the outcomes. Model discrimination was assessed by Area Under the Curve (AUC) of a Receiver Operating Characteristic (ROC). We computed 5000 simulations of the model using the sampling with replacement method to assess the generalisability of the models by comparing the bootstrapped c-index and confidence interval to the AUC obtained from the logistic model.

This data was analysed using Stata software package version 14.2, StataCorp, College Station, TX: StataCorp LP.

\section{Ethical Considerations}

Ethical approval for the Artesunate Versus Quinine in the Treatment of severe falciparum malaria in African children (AQUAMAT) study, registered under ISRCTN50258054, was obtained from each participating institutional or national ethics committee in addition to the Oxford Tropical Research Ethics committee (24). The use of data for this secondary analysis was approved by the Oxford-Mahidol research Unit Data Access Committee through an application for 'Datasets under the Custodianship of Mahidol Oxford Tropical Medicine Research Unit (MORU) Tropical Network'. The database used for this analysis was de-identified and anonymised.

\section{Results}

The baseline characteristics (Table 1) were collected at admission and there were no significant differences between the Artesunate and Quinine group.

Prognostic Factors Metabolic Acidosis 
In this study, $50.1 \%(2,296)$ of children presented with severe metabolic acidosis [base excess less than $8 \mathrm{mmol} / \mathrm{l}$ ] and there was no significant difference between males (48.9\%) and females (51.3\%). About a quarter, $24.1 \%$ had normal acid-base state and $25.8 \%$ had mild metabolic acidosis (BE: more than $8 \mathrm{mmol} / \mathrm{l}$ and less than $-3 \mathrm{mmol} / \mathrm{l}$ ). Among those who died, $82 \%$ (367), had severe metabolic acidosis. Survivors were $81 \%$ were less likely to have severe metabolic acidosis compared to those who died (OR = $0.19,95 \%$ Cl: $0.15-0.24$, p-value: < 0.001). Severe metabolic acidosis was present in $50.8 \%$ (1162) of patients in the artesunate arm and $49.5 \%$ (1134) of children in the quinine arm.

Table 2: Univariable and Multivariable Analysis of candidate predictors and severe metabolic acidosis

For the multivariable analysis (Table 2), patients from Kenya (OR: 4.86) and Rwanda (OR: 5.68) have significantly higher odds of presenting with metabolic acidosis. Every year increase in age was associated with a $22 \%$ decrease in likelihood of presenting with severe metabolic acidosis (OR: $0.78, \mathrm{Cl}$ : $0.75-0.81)$.

The clinical features with the strongest association to severe metabolic acidosis were deep breathing (OR: 5.41, Cl: 4.26-6.89), hypoglycaemia (OR: 5.22, Cl: 3.80-7.18) and acute kidney injury (BUN > 20 mg/dl) (OR: 3.99, Cl: 3.30-4.81).

In the multivariable analysis, children presenting with a coma were 1.79 times more likely to have severe metabolic acidosis when compared to those without coma (Cl: 1.36-2.35). Conversely, children who presented with convulsions were $21 \%$ less likely to have severe metabolic acidosis compared to those without convulsions (OR: 0.79, Cl: 0.65-0.95).

Children with respiratory distress (OR: 1.49, Cl: 1.21-1.83), prostration (OR: $1.64 \mathrm{Cl}: 1.30-2.03)$ and severe anaemia (OR: 1.40, Cl: 1.11-1.77) were more likely to have metabolic acidosis compared to those who did not have these signs.

Model performance is shown using a calibration plot in Fig. 2 of observed against predicted events plotted for 10 risk groups (c-index $=0.82$, slope $=1.1$ ). The Area Under the Curve (AUC) (Fig. 2) based on the final model was 0.81 (95\% Cl: $0.79-0.83$ ). The model has a positive predictive value (PPV) of $72.8 \%$ and a Negative Predictive value (NPV) of $72.3 \%$. The sensitivity and specificity plot cross at a probability cut-off of $44 \%$ where the sensitivity was $71.9 \%$ and the specificity was $73.1 \%$ (Fig. 4).

The model had good internal validation performed using bootstrapping with 5000 simulations (AUC = 0.81 (95\% Cl: 0.79-0.82).

Prognostic Factors of Acute Kidney Injury

Among children admitted with severe malaria in this study, 24.3\% (1110) had acute kidney injury. AKI was present among 217 participants (49.2\%) out of 441 who died (missing data on BUN for 86 results) and of the 4120 participants who survived, $21.7 \%$ (893) had AKI. Those who survived were $71 \%$ less likely to 
have AKI compared to those who died (OR: 0.29, Cl: 0.23-0.35, p-value: <0.001). The univariable analysis for factors associated with AKI are shown in the table 4.

After multivariable analysis (Table 4) and forward selection of predictors, factors associated with acute kidney injury upon admission were; increasing age (OR: 1.20, Cl: 1.15-1.25), coma (OR: 2.47, Cl: 1.783.42), prostration (OR: $1.52 \mathrm{Cl}$ : 1.14-2.02), decompensated shock (OR: 1.74, Cl: 1.15-2.63), black water fever (OR: 1.81, Cl: 1.22-2.69), jaundice (OR: $3.31 \mathrm{Cl}$ : 2.01-5.47), severe metabolic acidosis (OR: $4.02 \mathrm{Cl}$ : 3.30-4.89), mild anaemia (OR: 1.36, Cl: 1.05-1.76), severe anaemia (OR: 1.48, Cl: 1.11-1.96), hypoglycaemia (OR: 2.02 , Cl: 1.58-2.59), hypernatremia (OR: 5.74, Cl: 2.69-12.26) and hyperkalaemia (OR: 5.31. Cl: 4.15-6.80).

Figure 5 shows the model performance assessed using a calibration plot of observed against predicted events for 10 groups (c-index $=0.803$, slope $=0.9)$. The AUC ROC for the final model was $0.81(95 \% \mathrm{Cl}$ : $0.79-0.83$ ). The sensitivity and specificity plots (Fig. 7) cross at a probability cut-off of $25 \%$ where the sensitivity was $70.2 \%$ and the sensitivity was $77.7 \%$. The final model had a PPV of $50.1 \%$ and NPV of $89.1 \%$.

The model had good internal validation performed using bootstrapping with 5000 simulations AUC $=0.81$ (95\% Cl: 0.79-0.83).

\section{Discussion}

Although severe metabolic acidosis and acute kidney injury are major causes of death from severe malaria, diagnosing them is challenging in low resource settings. In this study, we identified clinical features of severe metabolic acidosis and acute kidney injury in children with severe malaria at admission. The models we describe can be used to predict a group of children with a high chance of having severe metabolic acidosis or acute kidney injury, which could then be selected for further laboratory work-up. Diagnosing these complications is important for the clinical management, monitoring, and, in the case of AKI, deciding on potential renal replacement therapy.

Severe Metabolic Acidosis

We found that the clinical features associated with severe metabolic acidosis in children with severe falciparum malaria at admission in this study were young age and prostration at admission. Clinical features that contribute to severe metabolic acidosis were severe anaemia, hypoglycaemia, acute kidney injury and black water fever. Features occurring because of severe metabolic acidosis were respiratory distress, deep breathing and coma at admission. All signs can be used to identify the group with a high probability of having severe metabolic acidosis.

Similar findings were identified in a prognostic study in the Gambia with comparable discriminative performance (ROC: 0.83) (17). This study identified; low Blantyre Coma Score (BCS: 0-2), high 
parasitaemia, high respiratory rate for age and deep breathing as clinical features associated with hyperlactataemia in children with severe malaria (17). Hyperparasitaemia was not associated with severe metabolic acidosis in our study and this may be attributed to the study in the Gambia occurring in a low transmission setting.

Signs of respiratory distress are commonly associated with Severe Metabolic Acidosis, as is the finding in this study and studies in the Gambia, Malawi and Kenya $(19,23,25,30)$. Despite the potential overlap between respiratory distress from severe malaria and severe pneumonia, we argue, in agreement with other studies, that respiratory distress is a major independent predictor of severe metabolic acidosis (3133).

Our study suggests that deep breathing is a strong independent predictor of severe metabolic acidosis as it is an important element of respiratory compensation as a consequence of metabolic acidosis (34). In this study, children with severe malaria presenting with deep breathing were 8 times more likely to have severe metabolic acidosis upon univariable analysis (OR: 8.74, Cl: 7.11-10.74) and 5 times more likely (OR: $5.41, \mathrm{Cl}: 4.26-6.89$ ) upon multivariable analysis. Although inter-observer variability may affect the use of this sign as a surrogate marker for Severe Metabolic Acidosis, it may be a reliable starting point for screening for children who require specialised management upon admission (35).

Our study found that younger children were more likely to have Severe Metabolic Acidosis upon admission with severe malaria. Although a study in South East Asia found that metabolic acidosis did not vary with age, our study findings are consistent with clinical trials in Tanzania $(36,37)$. This may suggest that the higher transmission rates of Malaria in Africa increase the odds of severe metabolic acidosis in children.

Mtove et al found that severe anaemia and hypoglycaemia were significantly associated with hyperlactataemia upon univariable logistic regression, as was the case in our findings (36). Severe anaemia is a known complication of malaria and contributes to fluid depletion and hypoxia in children with severe malaria and hypoglycaemia is a major cause of mortality in children with Malaria (9). These are complications of malaria that contribute to causing severe metabolic acidosis.

In contrast with our findings, Newton et al found deep breathing, coma, and hypoglycaemia to be highly specific for hyperlactatemia in three different countries (20). Their study concluded that these were not reliable surrogate markers of severe metabolic acidosis (20).

Our model performed well (AUC: 81) and, upon internal validation, deep breathing, hypoglycaemia, and coma were reliable prognostic predictors of severe metabolic acidosis. We recommend prospective, external validation studies to explore the generalisability of this model. To contribute to the improvement of the clinical management of these complications, further studies should study the effect of blood transfusions, fluid management and oxygen support to children identified as severe metabolic acidosis. 
We found that older children, and children who presented with coma, jaundice and hypoglycaemia were more likely to have AKI upon admission with severe malaria. Children who presented with decompensated shock, blackwater fever and anaemia (mild and severe) were more likely to have AKI as these are causes of AKI. Features associated with AKI as clinical consequences of AKI were, anuria, hypernatremia, hyperkalaemia and severe metabolic acidosis.

AKI is often underdiagnosed in children, and our observed prevalence of $24.3 \%$ among all admissions and $49.2 \%$ among all children who died is alarming. A study in the Gambia found that $25 \%$ of children with cerebral malaria had a glomerulo-tubular pattern of renal pathology (38). Although that study lacked the power to suggest a significant association between mortality and AKI in severe malaria, it is consistent with the strong significance of coma as an associated feature to AKI in our study (38).

Our analysis suggests that oliguria is not a reliable indicator of AKI in children with severe falciparum malaria. Only 7 of 5426 children had oliguria and although it was a significant sign at univariable analysis, it was not significant upon multivariable analysis. This may suggest that this is an inconsistent, or late sign of AKI as has been suggested in sub-Saharan Africa and India $(22,39)$.

While Black water fever was significantly associated with AKI, it was present in only 237 of 5426 children (4.4\%) (4). Children with severe falciparum malaria in India had an AKI incidence of $19 \%$ though none had black water fever (39). It remains an important feature, as it was the leading cause of AKI among children in a nephrology unit in the Democratic Republic of Congo (12).

Our findings of anaemia (mild and severe), decompensated shock, hypernatremia and hyperkalaemia being significantly associated with AKI are consistent with studies that identify the role of dehydration in the development of AKI in severe Malaria(12,38,39). Further study is needed to understand appropriate fluid management in children as fluid bolus resuscitation was associated with increased mortality in a large trial (40).

Our analysis suggests that severe metabolic acidosis and AKI are interrelated. Hypoglycaemia, coma and severe anaemia are features common to these two outcomes. This could reinforce these as major targets for clinical interventions

Increasing age being significantly associated with AKI in this study alludes to the changing epidemiology of severe malaria. An observational study in Kenya found a larger increase in mean age of severe malarial anaemia and cerebral anaemia between 1989 and 2016, when compared to children that did not have malaria (31). A higher mean age of renal involvement in children with severe malaria was seen in the DRC (6.7 years), India (7.7 years) and south-east Asia $(2,12,39)$. This is attributed to a delay of acquired immunity and increase of severe malaria in older children as malaria prevention efforts intensified for younger children over the years.

Our model performed well in distinguishing AKI by its presenting features. However, because of the high mortality among children with AKI and significance of late signs, it may be important to invest in 
biomarker diagnoses for AKI in African hospitals. This would allow for clinical staging of AKI which we were not able to do in this study.

\section{Limitations}

We conducted a retrospective analysis and could not follow up the clinical progress of severe metabolic acidosis or AKI for the study participants. There is a risk of inter-observer variation in identifying clinical signs which may over or under emphasize the presence of some features. Secondly, there is possible collinearity between AKI and Severe Metabolic Acidosis as there were clinical factors common to both. However, we were unable to explore the implications of the collinearity on presentation at admission or prognosis in children with severe malaria. Lastly, we did not test a previously developed model for severe metabolic acidosis because of the differences in measuring metabolic acidosis (36). Although we could not compare the performance of both models, the factors identified were similar to those in our study.

\section{Conclusions}

We conducted a secondary analysis of a large clinical trial spanning nine countries to identify prognostic features of severe metabolic acidosis and acute kidney injury in children with severe malaria. Identifying children who are likely to have these complications could help triage patients requiring further treatment or specialised therapy. Health policies should consider structures for early identification and treatment of these complications to reduce mortality from Malaria.

\section{Abbreviations}

AQUAMAT: Artesunate vs Quinine in the treatment of severe falciparum malaria in African children'. PPV: Positive Predictive Value, NPV: Negative Predictive Value, AUC: Area Under the Curve, ROC: Receiver Operating Characteristic, WHO: World Health Organisation, AKI: Acute Kidney Injury, SMA: Severe Metabolic Acidosis, aHBA: $a$-hydroxybutyric acid, $\beta H B A$ : $\beta$-hydroxybutyric acid, HPLA: $p$ hydroxyphenyllactic acid, BUN: Blood Urea Nitrogen, BE: Base Excess, OR: Odds Ratio, Cl: Confidence Interval, Hb: Haemoglobin, VIF: Variance Inflation Factor, BWF: Black Water Fever, BCS: Blantyre Coma Score

\section{Declarations}

Ethics approval: The Ethics approval for the AQUAMAT study for which the data of this study was obtained from the Oxford Tropical Research Ethics Committee and national ethics review boards of respective countries. The ethics approval for this study was obtained from the Oxford Tropical Research Ethics Committee and the approval to use the data for secondary analysis was obtained from the Mahidol Oxford Tropical Medicine Research Unit.

Consent to participate: Not Applicable 
Availability of data and materials: The datasets used and/or analysed during the current study are available from the corresponding author on reasonable request

Competing interests: The authors declare that they have no competing interests

Funding: This study was carried out in partial fulfilment of the requirements for the award of Master of Science in International Health and Tropical Medicine funded by the Oxford Weidenfeld and Hoffmann scholarship. The funding for research placement was supported by the University of Oxford, International Health and Tropical Medicine course. The AQUAMAT study was funded by the Wellcome Trust. The funders had no role in the study design, analysis, and manuscript writing.

Authors' contributions: The study concept was designed by MM, EO and GM. SL and KM provided feedback in refining the concept and scope of the study. The data analysis was done by GM, MM and EO. All authors provided feedback on the manuscript and approved the final version.

Acknowledgements: We thank the AQUAMAT trial group and the staff at the Mahidol Oxford Research Unit for their support during this study.

Authors Information: Conceptualization: GM, SL, AD, KM, EO and MM. Data curation: GM Formal analysis: GM, EO, MM. Funding acquisition for AQUAMAT: AD. The first manuscript draft was written by GM. All authors read and approved the final manuscript.

\section{References}

1. WHO. World malaria report 2018 [Internet]. Luxembourg, Geneva: Licence: CC BY-NC-SA 3.0 IGO; 2018. Available from: https://www.who.int/malaria/publications/world-malaria-report-2018/en/.

2. Dondorp AM, Lee SJ, Faiz MA, Mishra S, Price R, Tjitra E, et al. The Relationship between Age and the Manifestations of and Mortality Associated with Severe Malaria. Clin Infect Dis [Internet]. 10400(47):151-7. Available from: https://doi.org/10.1086/589287.

3. Kazembe LN, Kleinschmidt I, Sharp BL. Patterns of malaria-related hospital admissions and mortality among Malawian children: an example of spatial modelling of hospital register data. Malar J. 2006;10(5:93):1-10.

4. Seidlein L, Von, Olaosebikan R, Hendriksen ICE, Lee SJ, Adedoyin OT, Agbenyega T, et al. Predicting the Clinical Outcome of Severe Falciparum Malaria in African Children: Findings From a Large Randomized Trial. Clin Infect Dis [Internet]. 2012;54(8):1080-1090. Available from: https://doi.org/10.1093/cid/cis034.

5. Leopold SJ. Metabolomic characterisation of acidosis in severe falciparum malaria [Internet]. University of Oxford; 2018. Available from: https://ora.ox.ac.uk/objects/uuid:3a089f85-39dc-478f- 
b627-37accb5004ea/download_file?

file_format=pdf\&safe_filename=Leopold_DPhil_Thesis_Final_ORA.pdf\&type_of_work=Thesis.

6. Sriboonvorakul N, Ghose A, Hassan MMU, Hossain A, Faiz MA, Pukrittayakamee S, et al. Acidosis and acute kidney injury in severe malaria. Malar J [Internet]. 2018;12(128):1-8. Available from: https://doi.org/10.1186/s12936-018-2274-9.

7. Herdman MT, Sriboonvorakul N, Leopold SJ, Douthwaite S, Mohanty S, Hassan MMU, et al. The role of previously unmeasured organic acids in the pathogenesis of severe malaria. Crit Care [Internet]. 2015;19(1):1-11. Available from: http://dx.doi.org/10.1186/s13054-015-1023-5.

8. Nicholas J, White S, Pukrittayakamee TT, Hien M, Abul Faiz, Olugbenga A, Mokuolu AMD. Malaria. Lancet [Internet]. 2018;391(10130):1608-21. Available from: http://dx.doi.org/10.1016/\%0AS01406736(13)60024-0.

9. Maitland K, Newton CRJC. Acidosis of severe falciparum malaria: Heading for a shock? Trends Parasitol. 2005;21(1):11-6.

10. Patel H, Dunican C, Cunnington AJ. Predictors of outcome in childhood Plasmodium falciparum malaria. Virulence [Internet]. 2020;11(1):199-221. Available from: https://doi.org/10.1080/21505594.2020.1726570.

11. Thi T, Trang M, Phu NH, Vinh H, Waller DJ, White NJ. Acute Renal Failure in Patients with Severe Falciparum Malaria. 1992;874-80.

12. Aloni MN, Nsibu CN, Meeko-Mimaniye M, Ekulu PM, Bodi JM. Acute renal failure in Congolese children: A tertiary institution experience. Acta Paediatr Int J Paediatr [Internet]. 2012;101(11):514-8. Available from: https://ezproxy-prd.bodleian.ox.ac.uk:3688/doi/epdf/10.1111/j.16512227.2012.02827.x.

13. Esezobor Cl, Ladapo TA, Osinaike B, Lesi FEA. Paediatric Acute Kidney Injury in a Tertiary Hospital in Nigeria: Prevalence, Causes and Mortality Rate. PLoS One [Internet]. 2012;7(12). Available from: https://journals.plos.org/plosone/article/file?id=10.1371/journal.pone.0051229\&type=printable.

14. Maitland K, Kevin M, English M, Mithwani S, Peshu N, Marsh K, et al. Severe P. falciparum malaria in Kenyan children : evidence for hypovolaemia. QJM An Int J Med [Internet]. 2003;96(6):427-34. Available from: https://doi.org/10.1093/qjmed/hcg077.

15. Marsh K, Dayo F, Catherine W, Isaiah M, Maria W, Victoria M, Peter NCharles,W, Peter W, Peshu Norbert, Pasvol Geoffrey SR. Indicators of life-threatening malaria in african children. N Engl J Med [Internet]. 2019;332(21):1399-404. Available from:

https://www.nejm.org/doi/full/10.1056/NEJM199505253322102.

16. Dondorp AM, Chau TTH, Nguyen HP, Mai NTH, Pham PL, Ly VC, et al. Unidentified acids of strong prognostic significance in severe malaria. Crit Care Med. 2004;32(8):1683-8.

17. Bhaskaran K, Ebonyi AO, Walther B, Walther M. Predictors of hyperlactataemia among children presenting with malaria in a low transmission area in The Gambia. Malar J. 2013;12(423):1-11.

18. Crawley J, English M, Waruiru C, Mwangi I, Marsh K. Abnormal respiratory patterns in childhood cerebral malaria. Trans R Soc Trop Med Hyg. 1998;92(3):305-8. 
19. Taylor TE, Borgstein A, Molyneux ME, Elizabeth Q, Hospital C, Lansing E, et al. Acid-base status in paediatric Plasmodium falciparum malaria. 1993;99-109.

20. Newton CRJC, Valim C, Krishna S, Wypij D, Olola C, Agbenyega T, et al. The Prognostic Value of Measures of Acid/Base Balance in Pediatric Falciparum Malaria, compared with other clinical and laboratory measures. Clin Infect Dis. 2005;41(7):948-57.

21. Conroy AL, Hawkes M, Elphinstone RE, Morgan C, Hermann L, Barker KR, et al. Acute kidney injury is common in pediatric severe malaria and is associated with increased mortality. Open Forum Infect Dis. 2016;3(2):1-9.

22. Olowu WA, Niang A, Osafo C, Ashuntantang G, Arogundade FA, Porter J, et al. Outcomes of acute kidney injury in children and adults in sub-Saharan Africa: a systematic review. Lancet Glob Heal [Internet]. 4(4):e242-50. Available from: http://dx.doi.org/10.1016/S2214-109X(15)00322-8.

23. English M, Waruiru C, Amukoye E, Murphy S, Crawley J, Mwangi I, et al. DEEP BREATHING IN CHILDREN WITH SEVERE MALARIA: INDICATOR OF METABOLIC ACIDOSIS AND POOR OUTCOME. 1996;55(5):521-4.

24. Dondorp AM, Fanello Cl, Hendriksen IC, Gomes E, Seni A, Chhaganlal KD, et al. Artesunate versus quinine in the treatment of severe falciparum malaria in African children (AQUAMAT): An open-label, randomised trial. Lancet [Internet]. 2010;376(9753):1647-57. Available from: http://dx.doi.org/10.1016/S0140-6736(10)61924-1.

25. ENGLISH M, SAUERWEIN R, WARUIRU C, MOSOBO1 JO M. MARSH BLK. Acidosis in severe childhood malaria. Qjm [Internet]. 2002;90(9):601-4. Available from: https://www.ncbi.nlm.nih.gov/pubmed/9307760.

26. Plewes K, Turner GDH, Dondorp AM. Pathophysiology, clinical presentation, and treatment of coma and acute kidney injury complicating falciparum malaria. Curr Opin Infect Dis [Internet]. 2018;31(1):69-77. Available from: https://www.ncbi.nlm.nih.gov/pmc/articles/PMC5768231/.

27. Chellappan A, Bhadauria DS. Acute kidney injury in malaria: An update. Clin Queries Nephrol [Internet]. 2016;5(1):26-32. Available from: http://dx.doi.org/10.1016/j.cqn.2016.04.004.

28. Barros Pinto MP, Marques G. Severe malaria. Trop Med Int Heal [Internet]. 2020;48(1):143-6. Available from: https://onlinelibrary.wiley.com/doi/pdf/10.1111/tmi.12313_2.

29. Textor J, Zander B, Gilthorpe MS, Liskiewicz M. GTE. Robust Causal Inference using directed acyclic graphs- The R package dagitty.pdf [Internet]. 2017. Available from:

https://academic.oup.com/ije/article/45/6/1887/2907796.

30. Jallow M, Casals-pascual C, Ackerman H, Walther B, Walther M, Pinder M, et al. Clinical Features of Severe Malaria Associated with Death: A 13-Year Observational Study in The Gambia. 2012;7(9):1-8.

31. Njuguna P, Maitland K, Nyaguara A, Mwanga D, Mogeni P, Mturi N, et al. Observational study: 27 years of severe malaria surveillance in Kilifi, Kenya. 2019;1-9.

32. O'Dempsey TJD, McArdle TF, Laurence BE, Todd JE, Greenwood BM, Lamont AC. Overlap in the clinical features of pneumonia and malaria in african children. Trans R Soc Trop Med Hyg. 1993;87(6):662-5. 
33. English M, Punt J, Mwangi I, McHugh K, Marsh K. Clinical overlap between malaria and severe pneumonia in African children in hospital. Trans R Soc Trop Med Hyg. 1996;90(6):658-62.

34. Marsh K, Forster D, Waruiru C, Mwangi I, Winstanley M, Marsh V, et al. Indicators of life-threatening malaria in african children. N Engl J Med. 1995;332(21):1399-404.

35. English M, Murphy S, Mwangi I, Crawley J, Peshu N, Marsh K. Interobserver variation in respiratory signs of severe malaria. Arch Dis Child. 1995;72(4):334-6.

36. Mtove G, Nadjm B, Hendriksen ICE, Amos B, Muro F, Todd J, et al. Point-of-Care Measurement of Blood Lactate in Children Admitted With Febrile Illness to an African District Hospital. Clin Infect Dis [Internet]. 2011;53(6):548-554. Available from:

https://academic.oup.com/cid/article/53/6/548/357462.

37. Dondorp AM, Lee SJ, Faiz MA, Mishra S, Price R, Tjitra E, et al. The Relationship between Age and the Manifestations of and Mortality Associated with Severe Malaria. 2008;10400:151-7.

38. Weber MW, Zimmermann U, Hensbroek MB, Van, Frenkel J, Palmer A, Ehrich JHH, et al. Renal involvement in Gambian children with cerebral or mild malaria. 1999;4(5):390-4.

39. Prasad R, Mishra OP. Acute kidney injury in children with plasmodium falciparum malaria: Determinants for mortality. Perit Dial Int [Internet]. 2016;36(2):213-7. Available from: https://www.ncbi.nlm.nih.gov/pmc/articles/PMC4803368/.

40. Kathryn Maitland S, Kiguli MMed, Opoka RO, Engoru C, Olupot-Olupot P, Akech SO, Nyeko MB,ChB,R, Mtove MB,ChB,MMed,G, Reyburn H, Trudie Lang, Bernadette Brent JAE. Mortality after Fluid Bolus in African Children with Severe Infection. N Engl J Med [Internet]. 2011;364(26):2483-95. Available from: https://www.nejm.org/doi/full/10.1056/NEJMoa1101549.

\section{Tables}

Due to technical limitations, table 1 to 3 is only available as a download in the Supplemental Files section.

\section{Figures}




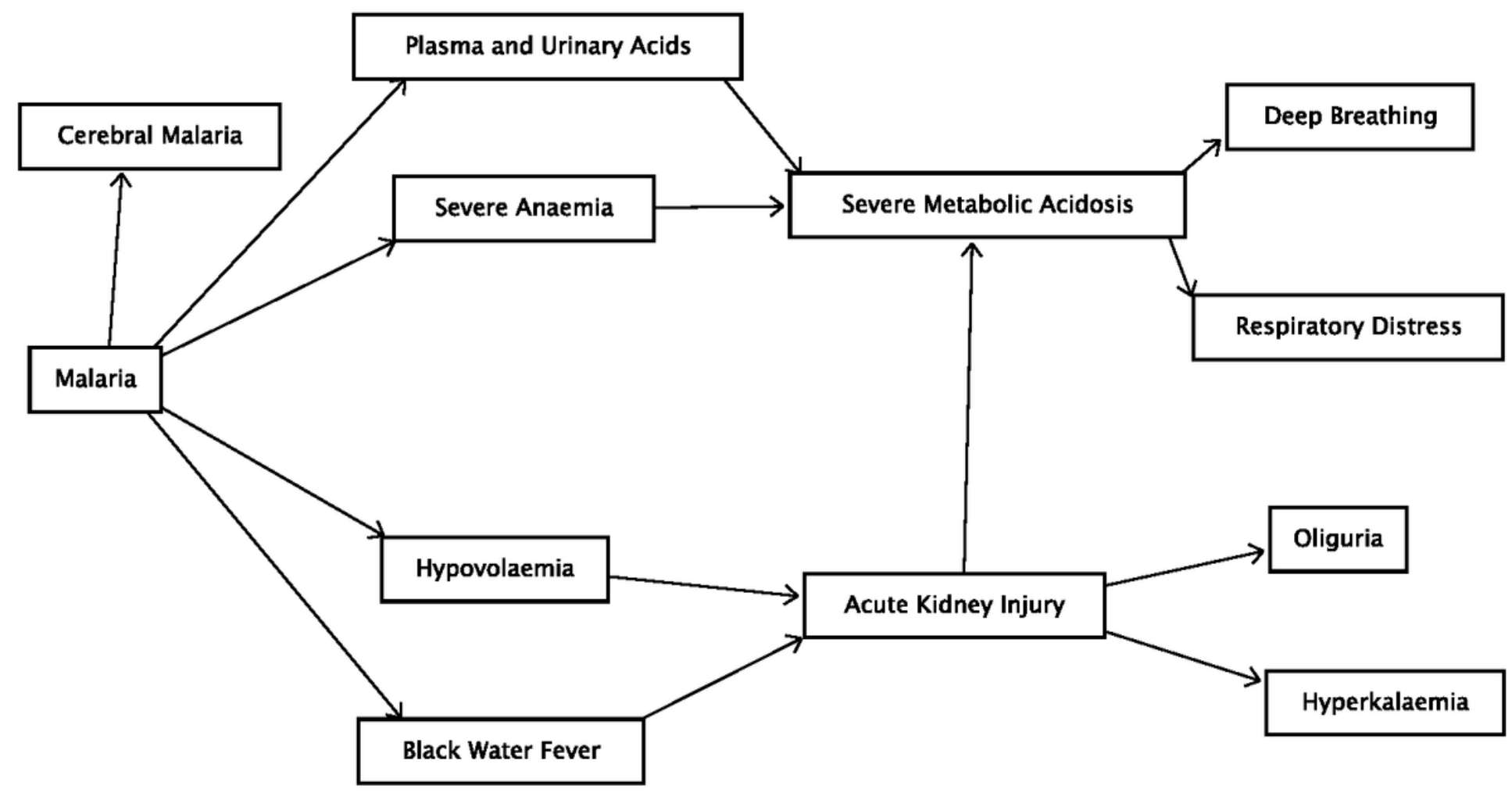

\section{Figure 1}

Conceptual Framework of Features associated with severe metabolic acidosis and acute kidney injury in children with Severe Falciparum Malaria (29) 


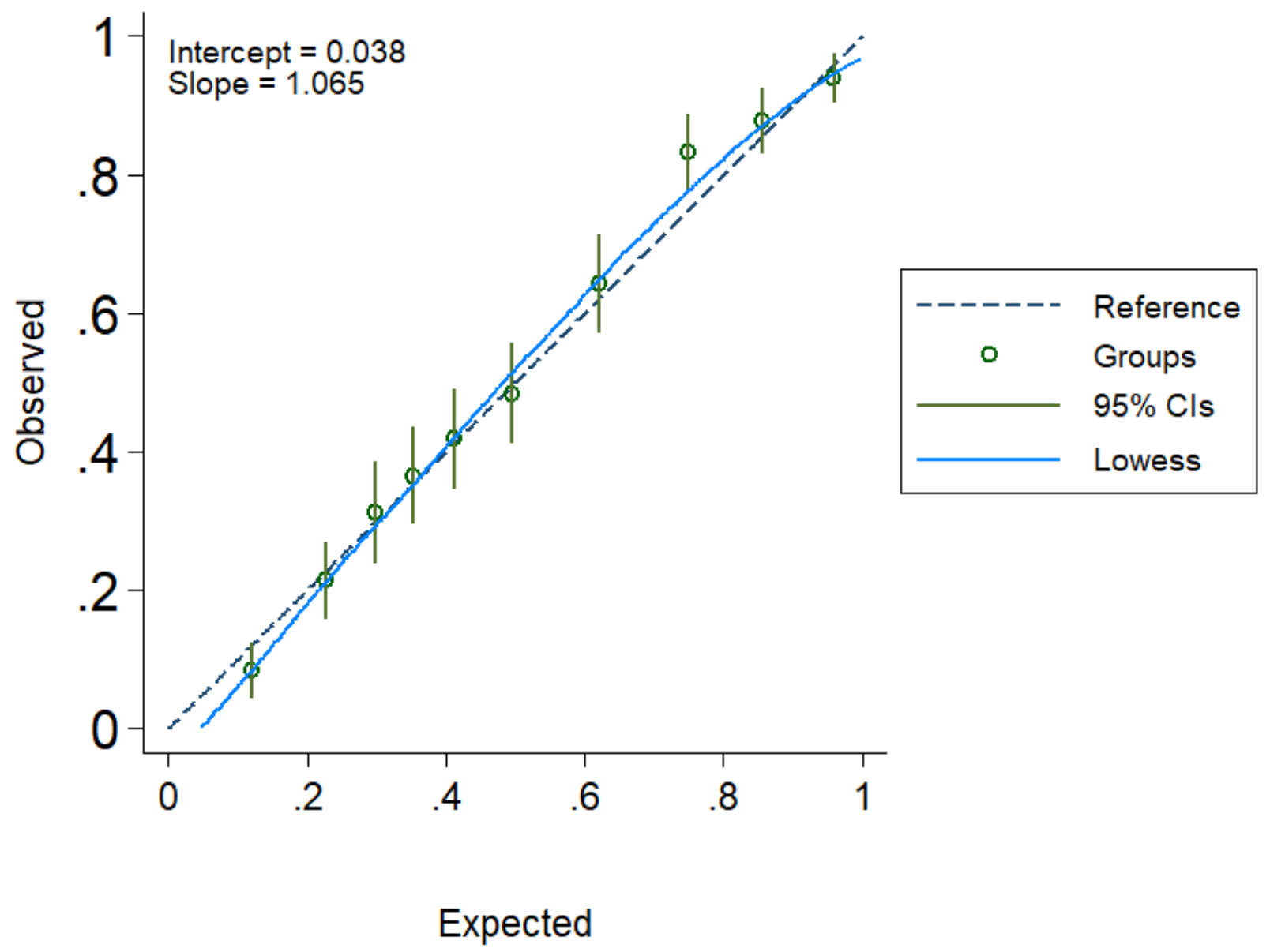

Figure 2

Calibration Plot for the Final Model of Factors Associated with Metabolic Acidosis 


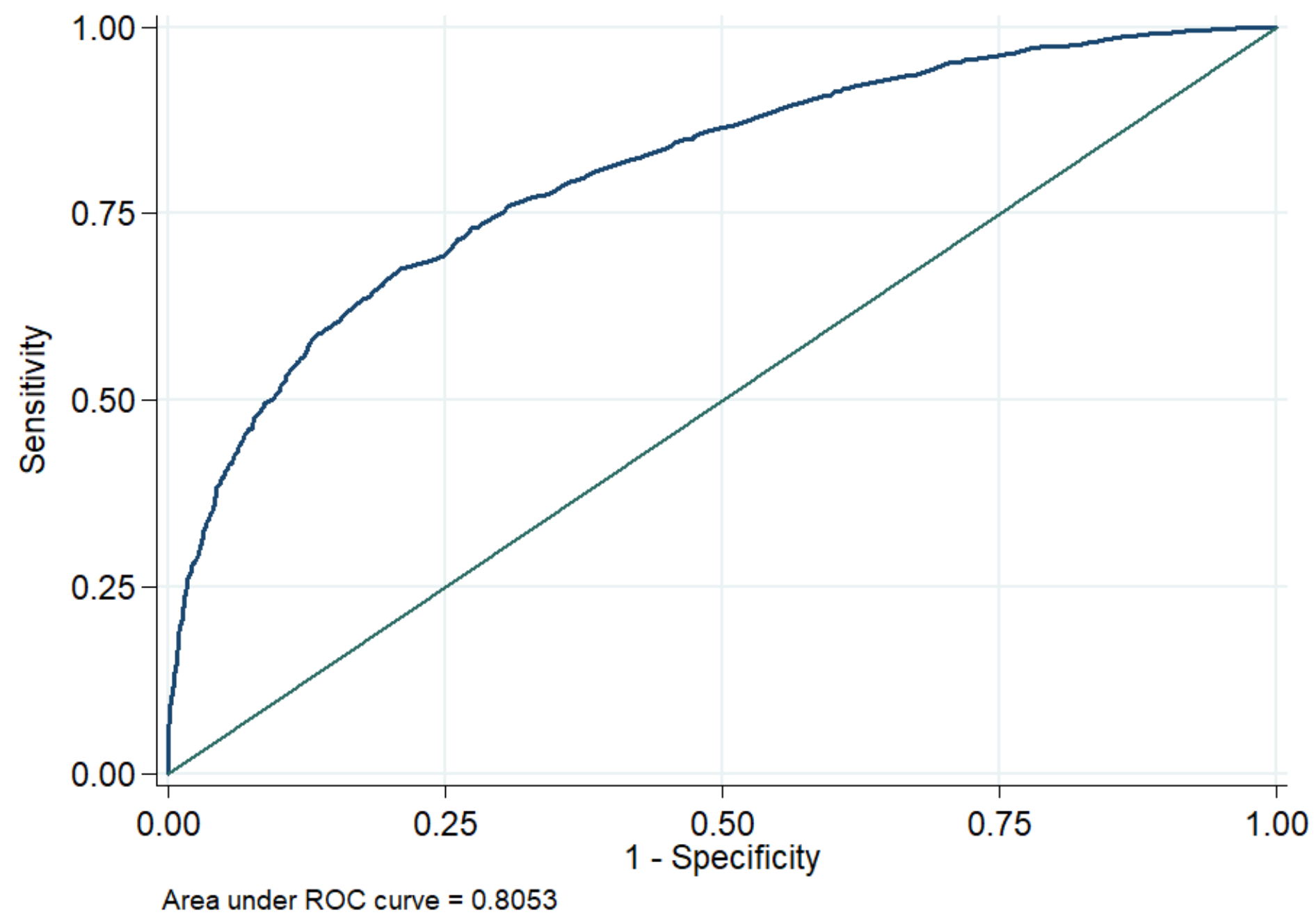

Figure 3

Receiver Operating Characteristic Curve for Final Model of Factors associated with Severe Metabolic Acidosis 


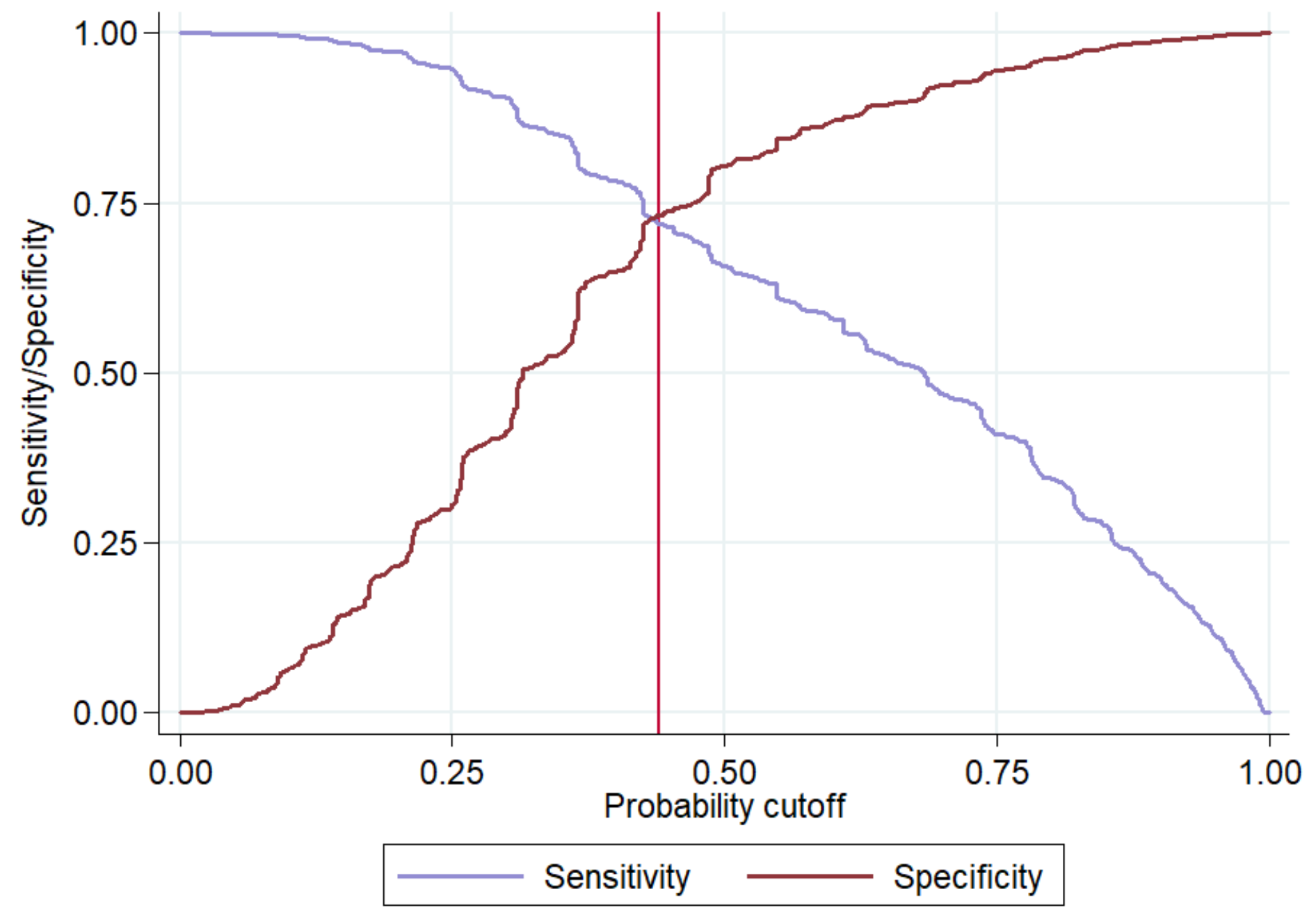

Figure 4

Sensitivity and Specificity Plot for Prognostic Model of Severe Metabolic Acidosis 


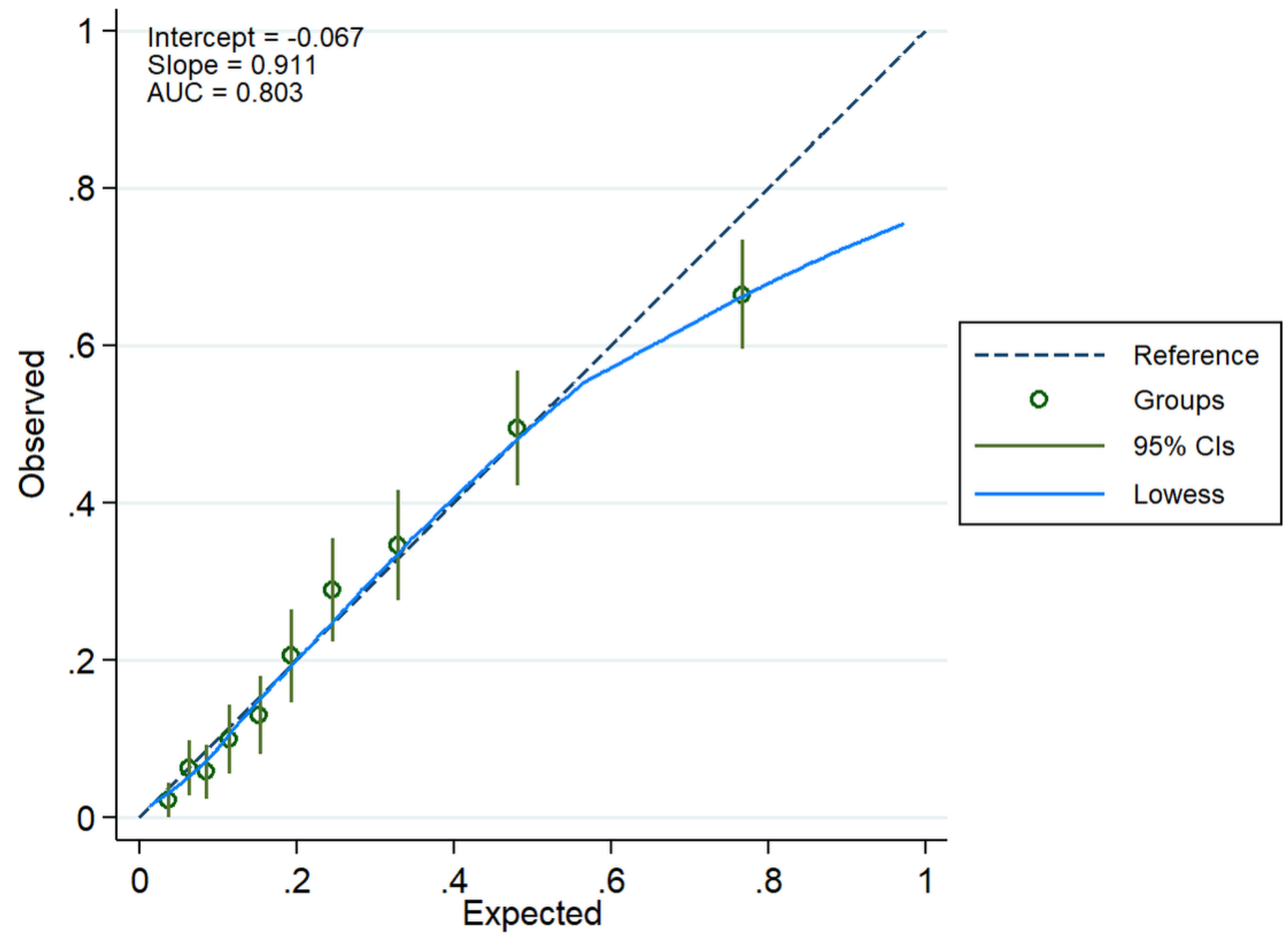

Figure 5

Calibration plot for the Final Model of Prognostic Factors Associated with Acute Kidney Injury 


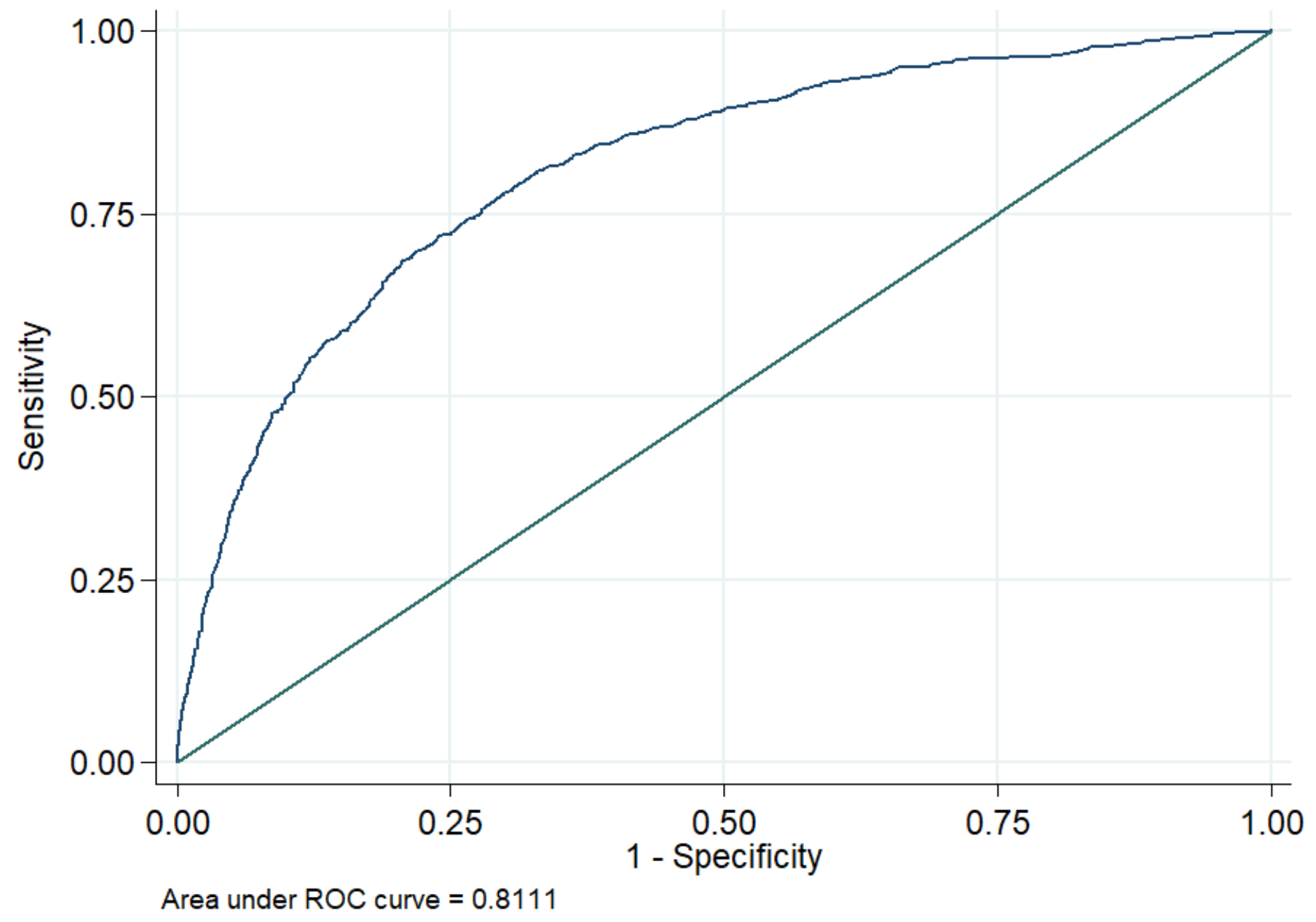

Figure 6

Receiver Operating Characteristic Curve for Model of Prognostic Factors of Acute Kidney Injury 


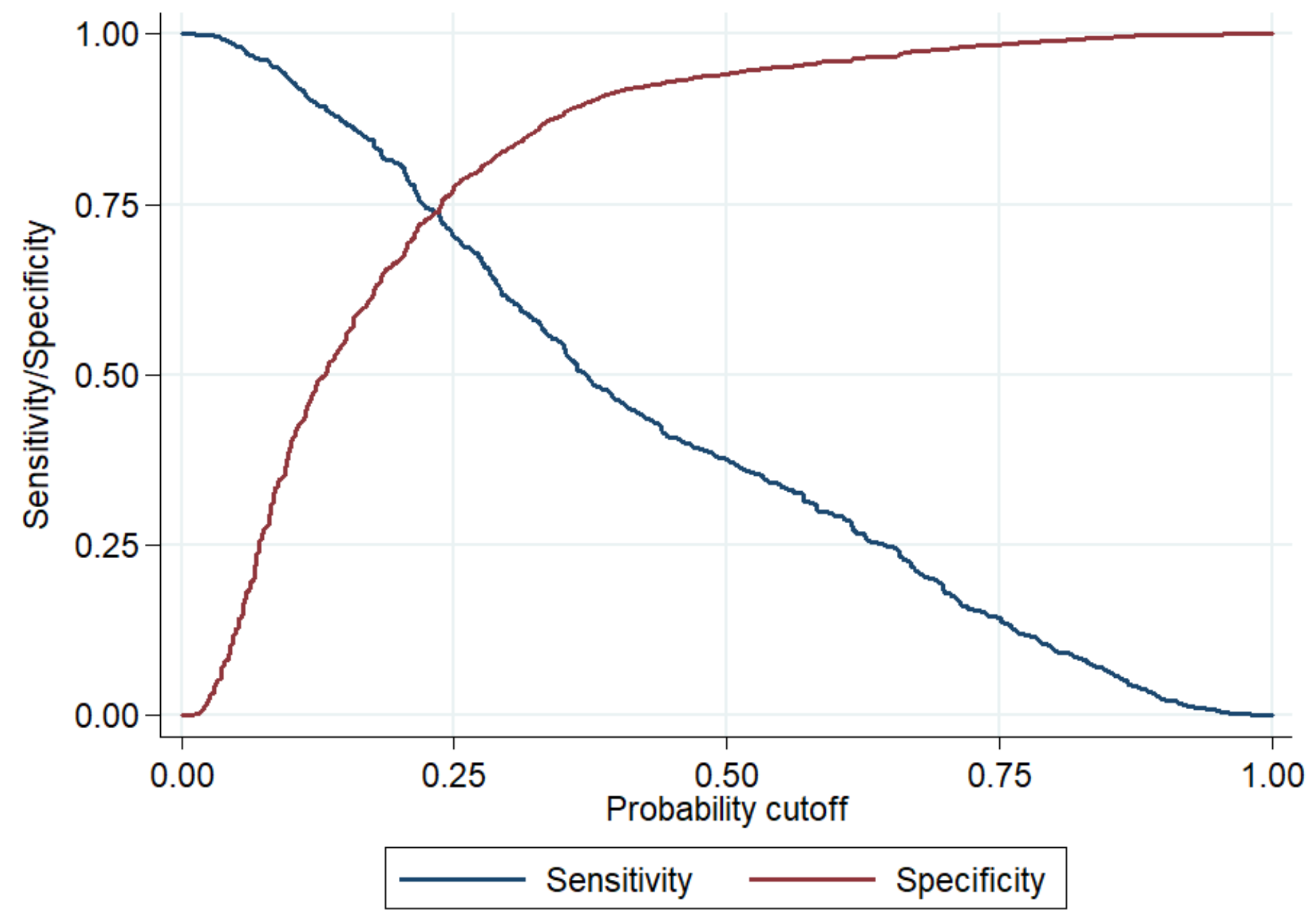

Figure 7

Sensitivity and Specificity plot for prognostic factors of acute kidney injury in children with severe malaria

\section{Supplementary Files}

This is a list of supplementary files associated with this preprint. Click to download.

- AdditionalFilesTablesforManuscript09012021.xlsx 